\title{
Theoretical assessment of transitions across thermionic, field, and space-charge-limited emission
}

\author{
Adam M. Darr, ${ }^{1}$ Caleb R. Darr, ${ }^{1}$ and Allen L. Garner (1) ${ }^{1,2,3, *}$ \\ ${ }^{1}$ School of Nuclear Engineering, Purdue University, West Lafayette, Indiana 47906, USA \\ ${ }^{2}$ School of Electrical and Computer Engineering, Purdue University, West Lafayette, Indiana 47907, USA \\ ${ }^{3}$ Department of Agricultural and Biological Engineering, Purdue University, West Lafayette, Indiana 47907, USA
}

(Received 27 December 2019; accepted 17 June 2020; published 24 July 2020; corrected 21 October 2020)

\begin{abstract}
As electron emission devices continue to push technological limits of device size, electric field, and temperature, characterization of device limitations due to thermionic (TE), field (FE), and space-charge-limited emission (SCLE) becomes increasingly important for device reliability and performance. While various theoretical studies have examined the transitions between any two of these mechanisms using asymptotic nexus theory and more detailed multiphysics solutions, a full assessment across all three regimes simultaneously using a single theory remains incomplete. Using a single-particle theory and the thermofield representation of current density, we derive equations that recover the asymptotic solutions for the Richardson-Laue-Dushman, Fowler-Nordheim, and Child-Langmuir laws for TE, FE, and SCLE, respectively. Various transitions are observed from this full solution, including TE to FE to SCLE, the Miram curve transitioning TE to SCLE, and the discovery of a field-enhanced Miram curve. Equating two of these asymptotic solutions yields a second-order nexus; a third-order nexus arises when all three asymptotic solutions match, yielding conditions for transitions from TE or FE to SCLE. We add Ohm's law and SCLE at pressure, modeled by the Mott-Gurney law, to nexus theory, generating diode parameter phase plots showing the areas of influence for all five mechanisms. This provides additional insight into mechanistic transitions to elucidate experimental results and guide system design under more extreme design requirements.
\end{abstract}

DOI: 10.1103/PhysRevResearch.2.033137

\section{INTRODUCTION}

Electron emission plays a critical role in diode physics, electron sources, and high power microwaves [1,2]. The primary electron emission mechanisms include thermal-, field, photo-, secondary-, and ferroelectric emission [1]. While most electron emission studies focus on vacuum [1-4] more recent studies have examined electron emission mechanisms for their contribution to gas breakdown for microscale gaps at pressures up to atmospheric [5,6]. These studies unify field emission (FE), often modeled by the Fowler-Nordheim (FN) equation [7], with Townsend avalanche [8-12], recovering the well-known Paschen's law [13] at sufficiently large gaps and ionization [11,12]. Matched asymptotic analyses have derived closed-form solutions assessing the transition from FE to Townsend avalanche and Paschen's law under various pressures, gap distances, and electrode conditions [9-12], analytically showing that breakdown voltage in the FE-driven regime decreases linearly with decreasing gap distance as a function of FE parameters $[12,14,15]$. Other studies have explored the impact of thermionic emission (TE) and thermofield emission on microscale gas breakdown [16-19]. Even at atmospheric pressure, reducing gap distances to submi-

\footnotetext{
*Corresponding author: algarner@purdue.edu

Published by the American Physical Society under the terms of the Creative Commons Attribution 4.0 International license. Further distribution of this work must maintain attribution to the author(s) and the published article's title, journal citation, and DOI.
}

croscale results in the transition from FE to space-chargelimited emission (SCLE) either by the Child-Langmuir (CL) law, which defines SCLE in a vacuum, or by the Mott-Gurney (MG) law, which defines SCLE with collisions [20]. SCLE plays a critical role in nanoscale electron emission devices [21]. Thus, characterizing electron emission at vacuum and nonvacuum pressures is critical for device reliability for applications ranging from vacuum electronics [1] to microelectromechanical systems (MEMS) and nanoelectromechanical systems (NEMS) [22,23].

Numerous studies have examined the transitions between various electron emission mechanisms, such as FE to SCLE [3,4,21,24,25], FE to TE [21,26-31], TE to photoemission [31], and TE to SCLE [32,33]. More recent studies have examined the unification of multiple mechanisms with the ultimate objective of unifying the theory for electron emission across a wide range of physical parameters, such as models unifying FE, TE, and photoemission [30,34,35]; FN, CL, and MG [20]; and FN, CL, MG, and Ohm's law (OL) with the addition of a series resistor [36]. Including more mechanisms increases complexity: thus, recent studies have introduced the framework of nexus theory, which equates the relevant asymptotic emission laws to characterize transition regions between mechanisms within an order of magnitude $[20,36]$. Understanding these mechanisms and the transitions between them is important for many key technologies, including photovoltaic cells, semiconductors, thermionic energy converters, and arc cathodes [29,31,37-39].

In this study, we focus on a fully analytic assessment of the transitions between TE, FE, and vacuum SCLE in a onedimensional, planar diode. Individually, TE is modeled by the 
Richardson-Laue-Dushman (RLD) equation [40]; FE by the FN equation [7]; and vacuum SCLE by the CL equation [1]. Experiments show enhanced emission during the transition between TE and FE above the predictions of either theory alone [41]. The link between TE and FE depends upon many material parameters such as surface treatments, impurities, work function, and field enhancement [42-47]. Even the precise shape of the potential barrier or work function has a significant effect [28,48-51]. The General-Thermal-Field (GTF) model [30,34,35], leverages the Murphy-Good approximations of key elliptic integrals [52,53] to formulate a TEFE model with excellent analytic behavior in the transition region. SCLE is modeled from first principles by the Poisson equation and can be solved in a variety of ways, including direct integration, transit time and capacitor models, and variational calculus $[1,54]$. To demonstrate the simplicity and flexibility of nexus theory, we fully incorporate our previous nexus work, which included OL and MG [20,36] with the FNRLD-CL nexuses. To summarize the objective of this paper, we study transitions among fundamental electron emission physics (FE, TE, SCLE) through the lens of models ranging from semiempirical (FN, RLD, GTF, MG) to fully analytic (CL, OL). An exact solution from first principles and GTF will be shown to recover the FN, RLD, and CL equations, while asymptotic nexus theory will be used to predict the relevant physics over a given physical parameter space by equating any/all of the models.

Section II outlines the derivation of the exact solution from single-particle theory, the asymptotes for the various mechanisms under appropriate limits, and nexus theory, which arises from equating two or more asymptotes. Section III applies this theory to various conditions to demonstrate the requirements for achieving different order nexuses and show the relationship between full and nexus theories. We provide concluding remarks in Sec. IV.

\section{THEORY}

Analogous to prior theoretical studies examining the transition from FE to SCLE for a planar diode [3,20], we consider the motion of a single electron emitted from the cathode at $x=0$, where $x$ represents position across the gap, with the anode at $x=D$ biased to an electrostatic potential $\phi(D)=V$. Poisson's equation in one-dimensional, Cartesian coordinates is

$$
\frac{d^{2} \phi}{d x^{2}}=\frac{\rho}{\epsilon_{0}},
$$

where $\rho$ is electron charge density, and $\epsilon_{0}$ is vacuum permittivity. The energy balance for a single electron emitted from the cathode with initial velocity $v_{i}$ is

$$
\frac{1}{2} m v^{2}=e \phi+\frac{1}{2} m v_{i}^{2},
$$

where $m, e$, and $v$ are electron mass, charge, and velocity, respectively. We assume the initial velocity to be the average of Maxwell-Boltzmann distribution, $(1 / 2) m v_{i}^{2}=(1 / 2) k_{B} T$, where $k_{B}$ is the Boltzmann constant, and $T$ is the cathode temperature. This assumption holds whenever space charge is present or the electric field is large [24], conditions which are fulfilled for all of the transitions considered in this study. The continuity equation is

$$
J=\rho v,
$$

where $J$ is the electron current density. Unlike prior SCLE to FE studies, we close the model by defining $J$ using the general-thermal-field (GTF) model, which includes temperature and surface field effects [35], rather than FN [3,20,36]. Including GTF and initial velocity differs from previous studies, which only included field and space charge [3,4,24,25], incorporated pressure [20], or added an external resistor in series with the gap [36,55]. For any $F \equiv e|E|$ and $T$, the general-thermal-field (GTF) equation models $J$ as

$$
J_{\mathrm{GTF}}(F, T)=A_{\mathrm{RLD}} T^{2} N(n, s),
$$

where $A_{\mathrm{RLD}}=\left(e m k_{B}^{2}\right) /\left(2 \pi^{2} \hbar^{3}\right), \hbar$ is the reduced Planck constant, $n \equiv \beta_{T} / \beta_{F}, \beta_{T}=1 /\left(k_{B} T\right)$, and $\beta_{F}, s$, and $N(n, s)$ are complicated functions of $F$ and $T$ (see the Appendix) [35].

To generalize the theory, we transform to dimensionless variables by defining

$$
\begin{aligned}
& J=J_{0} \bar{J} ; E=E_{0} \bar{E} ; x=x_{0} \bar{x} ; t=t_{0} \bar{t} ; T=T_{0} \bar{T} ; \phi=\phi_{0} \bar{\phi} ; F=F_{0} \bar{F} ; v=v_{0} \bar{v} ; R=R_{0} \bar{R} \\
& \mu=\mu_{0} \bar{\mu} ; T_{0}=\frac{\Phi}{k_{B}} ; J_{0}=A_{\mathrm{FN}} E_{0}^{2} ; E_{0}=B_{\mathrm{FN}} ; x_{0}=\frac{e E_{0} t_{0}^{2}}{m} ; \phi_{0} \equiv E_{0} x_{0} ; t_{0}=\frac{\epsilon_{0} E_{0}}{J_{0}} \\
& F_{0} \equiv e E_{0} ; v_{0} \equiv \frac{x_{0}}{t_{0}} ; \mu_{0}=\frac{e \epsilon_{0}}{m A_{\mathrm{FN}} B_{\mathrm{FN}}} ; R_{0}=\frac{\phi_{0}}{J_{0} S},
\end{aligned}
$$

where $\Phi$ is the work function, $A_{\mathrm{FN}}$ and $B_{\mathrm{FN}}$ are asymptotic FN coefficients given by $A_{\mathrm{FN}}=e^{3} /\left(16 \pi^{2} \hbar \Phi\right)$ and $B_{\mathrm{FN}}=$ $\left(4 \sqrt{2 m \Phi^{3}}\right) /(3 \hbar e)[6,12]$, and $S$ is the emission area. Table I reports physical constants and FN coefficients for tungsten $(\Phi \approx 4.5 \mathrm{eV}$ ). We perform these theoretical calculations neglecting electron mobility $\mu$ and external series resistance $R$; adding these terms to (1)-(3) would add terms within the force law and circuit equation, respectively, as discussed previously $[20,36]$.
Combining (1)-(3) with (5) and transforming to the time domain as before $[3,20,36]$, we obtain

$$
\frac{d^{2} \bar{v}}{d \bar{t}^{2}}=\bar{J} .
$$

Integrating (6) twice with boundary conditions $\bar{v}(0)=\bar{v}_{i}$ and $\bar{v}^{\prime}(0)=\bar{E}$ yields

$$
\bar{v}(\bar{t})=\frac{1}{2} \bar{J}^{2}+\bar{E} \bar{t}+\bar{v}_{i} .
$$


TABLE I. Typical values of physical parameters.

\begin{tabular}{llc}
\hline \hline Symbol & \multicolumn{1}{c}{ Quantity } & Value \\
\hline$A_{\mathrm{FN}}$ & Fowler-Nordheim coefficient (at $4.5 \mathrm{eV})$ & $3.44 \times 10^{-7} \mathrm{~A} \mathrm{~V}^{-2}$ \\
$B_{\mathrm{FN}}$ & Fowler-Nordheim coefficient (at $4.5 \mathrm{eV})$ & $6.55 \times 10^{10} \mathrm{~V} \mathrm{~m}^{-1}$ \\
$e$ & Electron charge & $1.6 \times 10^{-19} \mathrm{C}$ \\
$m$ & Electron mass & $9.11 \times 10^{-31} \mathrm{~kg}^{-1}$ \\
$k_{B}$ & Boltzmann's constant & $1.38 \times 10^{-23} \mathrm{~J} \mathrm{~K}^{-1}$ \\
$\varepsilon_{0}$ & Permittivity of vacuum & $8.854 \times 10^{-12} \mathrm{~F} \mathrm{~m}^{-1}$ \\
$\hbar$ & Reduced Planck's constant & $1.05 \times 10^{-34} \mathrm{~J} \mathrm{~s}$ \\
\hline \hline
\end{tabular}

Integrating (7) with the boundary condition $\bar{x}(0)=0$ gives

$$
\bar{x}(\bar{t})=\frac{1}{6} \bar{J} \bar{t}^{3}+\frac{1}{2} \bar{E} \bar{t}^{2}+\bar{v}_{i} \bar{t} .
$$

Defining the dimensionless transit time $\bar{\tau}$ such that $\bar{x}(\bar{\tau}) \equiv \bar{D}$ where $\bar{D}$ is the dimensionless gap distance, (2) shows that $\bar{v}(\bar{\tau})=\left(2 \bar{V}+\bar{v}_{i}^{2}\right)^{1 / 2}$ where $\bar{V} \equiv \bar{\phi}(\bar{D})-\bar{\phi}(0)$ is the dimensionless gap bias voltage. Solving (7) at $\bar{t}=\bar{\tau}$ yields

$$
\bar{\tau}=\frac{\bar{E}}{\bar{J}} \xi,
$$

where $\xi=-1+\left[1+\left(2 \bar{J} / \bar{E}^{2}\right)\left(\left(2 \bar{V}+\bar{v}_{i}^{2}\right)^{1 / 2}-\bar{v}_{i}\right)\right]^{0.5}$. Substituting (9) into (8) at $\bar{t}=\bar{\tau}$ yields

$$
6 \bar{D}=\frac{\bar{E}}{\bar{J}} \xi\left[\frac{\bar{E}^{2}}{\bar{J}} \xi(\xi+3)+6 \bar{v}_{i}\right]
$$

Equation (10) gives $\bar{V}$ parametrically as $\bar{V}=\bar{V}(\bar{E}, \bar{T})$. The useful dimensionless relationship $\bar{F}=\bar{E}$ recasts (4) as

$$
\bar{J}_{\mathrm{GTF}}(\bar{E}, \bar{T})=\bar{T}^{2} N(n, s),
$$

which gives the $\bar{J}-\bar{V}$ curve parametrically in terms of $\bar{E}$ and $\bar{T}$. While this full solution is cumbersome, it can be simplified at appropriate limits to yield FN, RLD, and CL.

For $\bar{J} \ll 1$, substituting the binomial expansion of $\xi$ into (10) shows that $\bar{E} \equiv \bar{V} / \bar{D}$ for the lowest order term in $\bar{J}$, which corresponds to the condition of no space charge. For both vacuum [3] and collisional [20] cases, this means that $\bar{J}$ asymptotically follows either the FN or RLD law in this limit. Using the limits of $\bar{J}_{\mathrm{GTF}}$ [34], $\bar{E} \gg \bar{T}$ yields the FN limit where $\bar{J}=\bar{J}(\bar{V})$ and $\bar{T} \gg \bar{E}$ yields RLD with $\bar{J}=\bar{J}(\bar{T})$ [35]. Using (5), these two asymptotic emission equations are

$$
\begin{gathered}
\bar{J}_{\mathrm{FN}}=\bar{E}^{2} e^{-1 / \bar{E}}=(\bar{V} / \bar{D})^{2} e^{-\bar{D} / \bar{V}}, \\
\bar{J}_{\mathrm{RLD}}=\frac{9}{4} \bar{T}^{2} e^{-1 / \bar{T}},
\end{gathered}
$$

where the factor of $9 / 4$ in (13) arises due to the scaling factors defined in (5).

Considering the space-charge limit of $\bar{J} \gg 1$ yields a general CL (GCL) equation $\bar{J}_{\mathrm{GCL}}$. Expanding (10) in powers of $1 / \bar{J}$ gives

$$
\begin{aligned}
\bar{J}_{\mathrm{GCL}} & \approx\left(\frac{2}{9 \bar{D}^{2}}\right)\left(\sqrt{2 \bar{V}+\bar{v}_{i}^{2}}-\sqrt{\bar{v}_{i}^{2}}\right)\left(\sqrt{2 \bar{V}+\bar{v}_{i}^{2}}+2 \sqrt{\bar{v}_{i}^{2}}\right)^{2}=\bar{J}_{\mathrm{CL}}\left(\sqrt{1+\frac{\bar{v}_{i}^{2}}{2 \bar{V}}}-\sqrt{\frac{\bar{v}_{i}^{2}}{2 \bar{V}}}\right)\left(\sqrt{\left.1+\frac{\bar{v}_{i}^{2}}{2 \bar{V}}+2 \sqrt{\frac{\bar{v}_{i}^{2}}{2 \bar{V}}}\right)^{2}}\right. \\
& =\bar{J}_{\mathrm{CL}}\left[\left(1+\frac{\bar{v}_{i}^{2}}{2 \bar{V}}\right)^{3 / 2}-\left(\frac{\bar{v}_{i}^{2}}{2 \bar{V}}\right)^{3 / 2}+3 \sqrt{\frac{\bar{v}_{i}^{2}}{2 \bar{V}}}\right] \approx \bar{J}_{\mathrm{CL}}\left(1+3 \sqrt{\frac{\bar{v}_{i}^{2}}{2 \bar{V}}}\right)
\end{aligned}
$$

where $\bar{J}_{\mathrm{CL}} \equiv\left(4 \sqrt{2} \bar{V}^{3 / 2}\right) /\left(9 \bar{D}^{2}\right)$ is the CL equation [1] with $\bar{v}_{i}=0$, or $\bar{T}=0$. The final expression in (14) is obtained by keeping the lowest-order term of $\left[\bar{v}_{i}^{2} /(2 \bar{V})\right]^{1 / 2}$ in the space-charge limit of $\bar{V} \rightarrow \infty$; since this assumption is not always valid, we will use the penultimate expression in (14) when referring to GCL. For comparison, another study derived a form of the generalized CL equation as $\quad J_{\mathrm{GCL}}=J_{\mathrm{CL}}\left[\left(m v_{0}^{2} /(2 e V)\right)^{1 / 2}+\left(1+m v_{0}^{2} /(2 e V)\right)^{1 / 2}\right]^{3}$. Applying the definition from (5) gives $\bar{J}_{\mathrm{GCL}}=$ $\bar{J}_{\mathrm{CL}}\left[\left(\bar{v}_{i}^{2} /(2 \bar{V})\right)^{1 / 2}+\left(1+\bar{v}_{i}^{2} /(2 \bar{V})\right)^{1 / 2}\right]^{3}$, which also reduces to $\bar{J}_{\mathrm{GCL}} \approx \bar{J}_{\mathrm{CL}}\left(1+3\left(\bar{v}_{i}^{2} /(2 \bar{V})\right)^{1 / 2}\right)$ for large $\bar{V}$ [56]. For zero temperature, $\bar{v}_{i}=0$ and (14) reduces exactly to $\bar{J}_{\mathrm{CL}}$.

While the asymptotes defined in (12)-(14) adequately model $\bar{J}$ in their respective regimes, the full solution (10) is necessary near transition regions. Analogous to the transitions between $\mathrm{CL}$ and $\mathrm{FN}$ in vacuum [3] and $\mathrm{CL}, \mathrm{MG}$, and $\mathrm{FN}$ with collisions [20], the intersections of (12)-(14) show the general conditions for the transition between RLD, FN, and CL. Equating (12) and (13) shows that the FN to RLD transition occurs at $\bar{E} \approx \bar{T}$ for $\bar{T} \ll 1$ and $\bar{E} \approx 3 \bar{T} / 2$ for $\bar{T} \gg 1$. To demonstrate the physical relevance of these values, consider tungsten $(\Phi \approx 4.5 \mathrm{eV})$ at its melting temperature, $T=3695 \mathrm{~K}$, or $\bar{T}=0.071$. Tungsten has the highest melting point of any metal and a relatively high work function, thus we can safely assume $\bar{T} \ll 1$ for all metallic diodes and use the $\bar{E} \approx \bar{T}$ approximation for most physically relevant devices without loss of generality. For the FN to CL transitions, we first assume $\bar{E}=\bar{V} / \bar{D}$ in (12) based on the limit derived above for the case of no space charge. To assess the second term in the radical of (14) for the CL limit, consider a different high physical temperature limit of $T \approx 4000 \mathrm{~K}, \Phi \approx 4 \mathrm{eV}$. 
This gives $\bar{v}_{i}^{2} /(2 \bar{V})=1$ for $\bar{V}=1.86 \times 10^{-3}$ or $V=0.17 \mathrm{~V}$. Thus, for higher voltages corresponding to the CL limit, $\bar{v}_{i}^{2} /(2 \bar{V}) \ll 1$, which means we can ignore this term in (14) and use CL to obtain $\bar{D} \approx \bar{V} \ln [(9 \sqrt{\bar{V}}) /(4 \sqrt{2})]$. The FN to CL transition cannot be usefully simplified beyond equating (13) and (14); however, we can derive a third-order nexus when all three asymptotes match such that the FN-CL and FN-RLD lead directly to RLD-CL transitions. The RLD-FN-CL nexus (the ordering of terms is irrelevant) occurs when

$$
\frac{1}{\bar{T}_{N}}=\frac{1}{\bar{E}_{N}}=\frac{\bar{D}_{N}}{\bar{V}_{N}}=\ln \left[\frac{\left(9 \sqrt{\bar{V}_{N}}\right)}{(4 \sqrt{2})}\right]
$$

where the subscript $N$ denotes that the parameter describes a nexus point. An important property of (15) is that fixing any one of $\bar{T}_{N}, \bar{D}_{N}$, or $\bar{V}_{N}$ at the nexus fully defines the other two (and $\bar{E}$ in the non-SCLE limit); therefore (15) indicates when the full solution of (10) must be used to account for the interplay between emission mechanisms rather than the simpler models above. The full third-order nexus in (15) becomes second order (FN-RLD, FN-CL, or RLD-CL) by varying one of the parameters. This process must be used with care since these degraded nexuses retain the assumptions used to derive (15), such as $\bar{E}_{N} \approx \bar{T}_{N}$ and using CL instead of GCL from (14).

Nexus theory offers a powerful tool for design and simulation, especially in understanding whether a subset of parameters will be isolated from transition physics or not, motivating the use of asymptotic or much more complicated multiphysics solutions, respectively. The main benefit of nexus theory over full analytic-numerical models is that new theories can be added easily. For instance, we can add MG and OL asymptotic behavior from our previous work in nexus theory $[20,36]$ to (15), as

$$
\begin{gathered}
\bar{J}_{\mathrm{MG}}=\frac{9 \bar{\mu} \bar{V}^{2}}{8 \bar{D}^{3}}, \\
\bar{J}_{\mathrm{OL}}=\frac{\bar{V}}{\bar{R}},
\end{gathered}
$$

where $R$ is the resistance of an external series resistor, $S$ is the cathode emission surface area, and $\mu$ is electron mobility, inversely proportional to pressure $[20,36]$. Full solutions incorporating this physics may be derived by incorporating the new physics in (1)-(4) analogous to the earlier work that just included FE $[20,36]$.

The parameter space may be divided into five distinct physical regions, separated by transition curves (second-order nexuses) equating any permutation of (12)-(14), (16), and (17). Matching three or more asymptotes yields a third-order or higher nexus. Equations (16) and (17) permit generalization of (15) to a fifth order of nexus as

$$
\begin{aligned}
\frac{1}{\bar{T}_{N}} & =\frac{1}{\bar{E}_{N}}=\frac{\bar{D}_{N}}{\bar{V}_{N}}=\ln \left[\frac{\left.\left(9 \sqrt{\bar{V}_{N}}\right)\right]}{(4 \sqrt{2})}\right]=\frac{81 \bar{\mu}_{N}}{32 \sqrt{2 \bar{V}_{N}}} \\
& =\exp \left(-\frac{\bar{D}_{N}}{2 \bar{V}_{N}}\right) \sqrt{\frac{\bar{R}_{N}}{\bar{V}_{N}}} .
\end{aligned}
$$

One may use (18) to assess the proximity of a device's physical parameters to nexuses without resorting to using cumbersome exact solutions; if far from any transitions, simple (asymptotic) models may be used.

\section{RESULTS}

Figures 1(a)-1(c) show the transitions between mechanisms as $\bar{J}=\bar{J}(\bar{V})$ for fixed $\bar{D}=0.55$ and three fixed $\bar{T}$, including $\bar{T}_{N}=1.98$ for the FN-RLD-CL nexus, while Figs. 1(e) and 1(f) show the percentage match between the full solution and each asymptotic model, FN, RLD, and CL/GCL. The kinks in the FN and RLD contributions arise due to the transition of the piecewise continuous GTF model between several representations of the barrier potential [27,35]. To show a range of possible $\bar{J}-\bar{V}$ morphologies that may be relevant under different conditions, Fig. 1 spans many more orders of magnitude than possible for a single experiment. Since experiments have considered all these transitions piecemeal (FN-CL, FN-RLD, RLD-CL), different sections of Fig. 1 (or similar plots) will be relevant to experimentalists under appropriate conditions.

We also selected temperatures to clearly demonstrate possible morphologies. Taking tungsten again as an example, only the $\bar{T}=0.03 \bar{T}_{N}=0.06<0.071$ curve shown in Figs. 1(a) and 1(d) is at a physically realizable temperature for this particular gap distance. Indeed, (15) shows that $\bar{T}_{N}=0.071$ would require $\bar{D}>10^{11}$; for tungsten, (5) gives $x_{0}=1.8 \times 10^{-9} \mathrm{~m}$, making $D \gtrsim 180 \mathrm{~m}$. However, surface treatments can lower the work function of tungsten as low as $1 \mathrm{eV}$ [57], which reduces the dimensionless melting temperature to $\bar{T}_{m}=0.3184$, which only requires $\bar{D} \approx 10^{3}$; since $x_{0}$ also depends upon work function, we obtain $x_{0}=$ $8.5 \times 10^{-10} \mathrm{~m}$, which gives $D \approx 850 \mathrm{~nm}$. For a temperature as low as $1500 \mathrm{~K}(\bar{T}=0.129)$, the nexus gap distance is only $\bar{D}=1.62 \times 10^{7}$, or $1.37 \mathrm{~cm}$. This feasibility makes these types of materials prime candidates for studying how current varies with voltage when all three mechanisms are relevant.

For the lowest temperature, shown in Figs. 1(a) and 1(d)physically realizable for simple metallic tungsten diodes-we observe clear transitions from RLD to FN to CL, with CL and GCL coincident since $\bar{V} \gg \bar{T}$. Two second-order nexuses occur at $\bar{T}=\bar{E} \equiv \bar{V} / \bar{D}$ and $\bar{D}=\bar{V} \ln (9 \sqrt{\bar{V}} /(4 \sqrt{2}))$, or $\bar{V}=$ 0.033 and 1.09 , respectively. Figure 1 (d) demonstrates the importance of these nexuses. Only at very high $\bar{V}$ does the full solution match within $20 \%$ of any asymptote for any significant range of $\bar{V}$. An extended section where $\tilde{J} \approx$ $\tilde{J}_{\text {RLD }}$ occurs for the intermediate temperature $T=0.15 T_{N}$ due to the higher $\bar{V}(\sim 0.165)$ for the RLD-FN nexus. This sort of analysis would allow a researcher to confidently use the simple RLD model for $\bar{V}<0.005$, or 0.03 $\mathrm{V}$ for the low work-function tungsten example. Figures 1(b) and 1(e) show that increasing $\bar{T}$ causes $\bar{J}$ to follow RLD for a much longer range of $\bar{V}$ before transitioning to FN and GCL.

Curiously, for the nexus case in Figs. 1(c) and 1(f), we see what looks like a GCL region below the nexus and CL above. Figure 1 was generated parametrically varying $\bar{E}$. As $\bar{E} \rightarrow 0, \bar{V} \rightarrow 0.15$, which accounts for the seemingly missing 



FIG. 1. Dimensionless current density $\bar{J}$ as a function of dimensionless gap voltage $\bar{V}$ at fixed dimensionless gap distance $\bar{D}=0.55$ for various nondimensional temperatures (a) $\bar{T}=0.03 \bar{T}_{N}$, (b) $0.15 \bar{T}_{N}$, and (c) $\bar{T}_{N}$, where $\bar{T}_{N}=1.98$ is the nexus between Fowler-Nordheim (FN) Richardson-Laue-Dushnman (RLD), and Child-Langmuir (CL) from (15). The dotted boxes in (a) and (b) correspond to the parameter space of (c) for comparison. The asymptotic field emission (black line, FN), thermal emission (green dashes, RLD), and space-charge-limited emission (blue dot-dashed line, CL, and blue dotted line, generalized CL or GCL) limits are calculated from (12)-(14), and compared to the exact solution (10), (11) (red boxes). The exact solution follows the highest $\bar{J}$ from emission laws (RLD or FN) until reaching the space-charge emission limit (CL or GCL) at high $\bar{V}$. Panels (d)-(f) divide the full solution by each asymptote for (a)-(c), respectively, for the relevant transition ranges of $\bar{V}$. These subfigures only show clear deviations of the full solution, hence the smaller range of $\bar{V}$ in (c) and (f) than the remaining panels.

RLD region in the high-temperature plots-the temperature was so high that the baseline thermal current, combined with the initial velocity term, only allows for high $\bar{V}$ solutions to (9) and (10). Figure 1(c) shows that the nexus occurs when the asymptotes for FN, RLD, and CL all match. We hypothesize that the reason for the GCL $\rightarrow$ Nexus $\rightarrow$ CL morphology is that RLD leads to space charge at lower $\bar{V}$, but once $\bar{E}$ increases enough, the initial velocity effect is subsumed by the large amount of acceleration. Then, since $\bar{V} \gg \bar{T}$ above the nexus, CL once again becomes the appropriate SCLE model since at high $\bar{V}$ GCL matches asymptotically with CL.

The matching of the asymptotes with the full solution differs dramatically from our previous results with a FN-MGCL nexus, which had only $40 \%$ agreement at the nexus [20]. We hypothesize that the full solution undershot the previous
FN-MG-CL nexus so much since the single FE model, FN, was more strongly depressed by two SCLE mechanisms (CL and MG equations), which limit emission [20]. MG also includes a friction term in the energy balance [20], whereas (4) exhibits no loss. The RLD-FN-CL nexus reported here considers two emission mechanisms, FE and TE (FN and RLD models, respectively), which are better able to compensate for a single SCLE limiting mechanism modeled by CL. Transitions exert a significant influence over several orders of magnitude of $\bar{V}$ and $\bar{J}$. Although experiments operate over a much smaller parameter space, understanding the full range of possible physics indicates the conditions that require a more refined model, even relatively far from transition physics.

When voltage is fixed, the tendency of the full solution to transition from TE to SCLE at various $\bar{V}$ depending upon $\bar{T}$ 

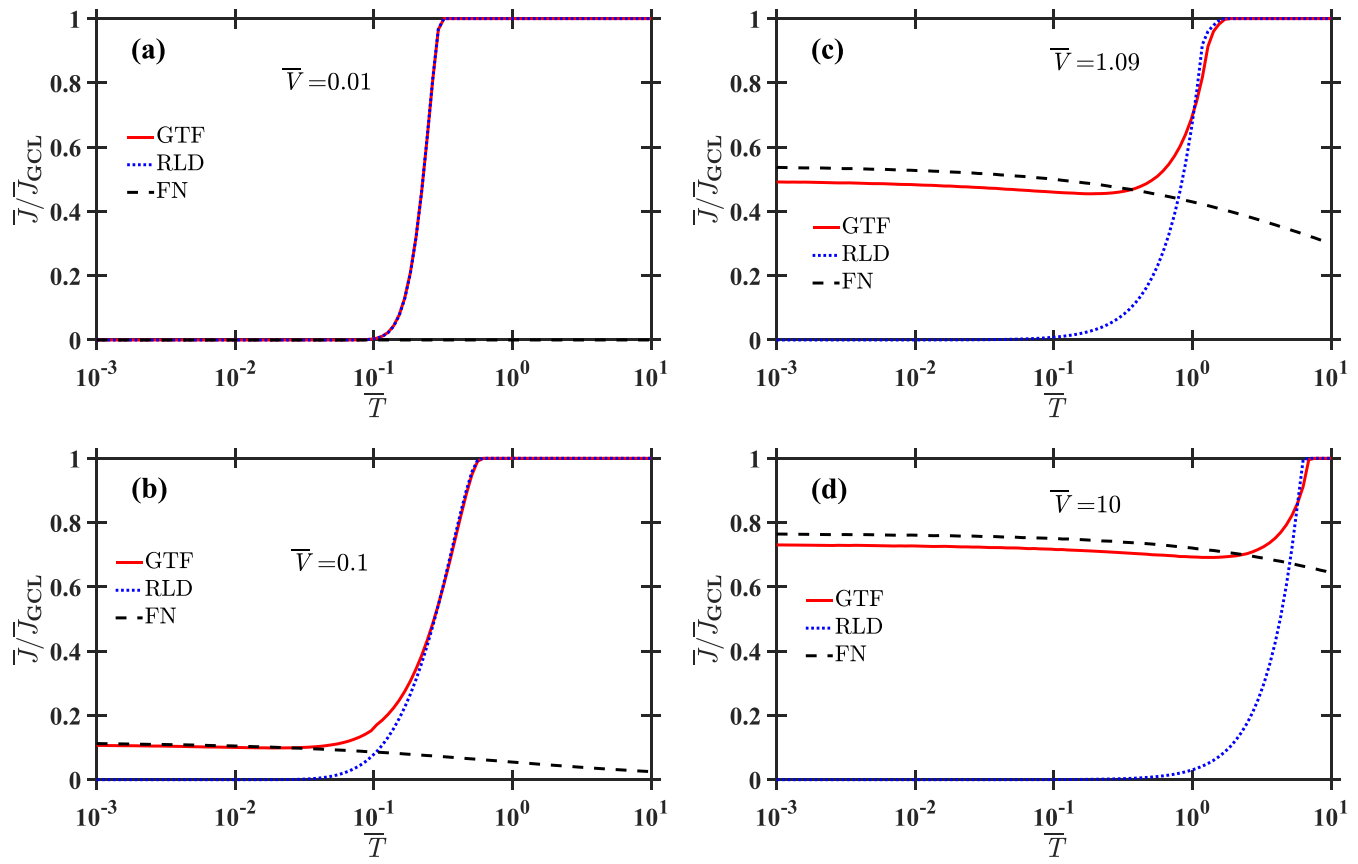

FIG. 2. Field enhanced Miram curve at constant dimensionless gap distance $\bar{D}=0.55$, showing the ratio between the full solution from (10), (11) and the generalized Child-Langmuir (GCL) law (14) as a function of dimensionless temperature $\bar{T}$. The standard Miram curve gradually transitions to unity at high $\bar{T}$, but a field contribution at high dimensionless voltage $\bar{V}$ gives a baseline current contribution and softens the knee where the behavior transitions to space charge. (a) Traditional Miram curve. (b) Slight field enhancement at low temperatures. (c) The nexus $\bar{V}$ causes significant field emission and a soft knee at transition to GCL. (d) Near-space-charge levels of field contribution leading to a higher temperature transition to space-charge-limited emission.

is well understood as a normalized rolloff or Miram curve [32,33]. Figure 2 shows various Miram curves at several fixed $\bar{V}$ and $\bar{D}=0.55$ to match with Fig. 1. All parts of Fig. 2 were calculated by simultaneously solving (9) and (10) with the GTF, FN, and RLD models from (11)-(13). In the FN and RLD regions, $\bar{E}$ was varied until the correct fixed $\bar{V}$ was found; however, once the solution transitioned fully to space charge at higher $\bar{T}$, there was no solution and the ratio was assumed to be unity, hence the sharp kink at the end of the knee. Other GCL equations exist which have lower current, so experimentalists are advised to generate field-enhanced Miram curves with the GCL model that best fits their assumptions [56].

Figure 2(a) resembles a standard Miram curve; however, Figs. 2(b)-2(d) show that this behavior can be modified by FE providing a baseline current density, resulting in a field enhanced Miram curve with a softer knee. These new field enhanced Miram curves transition first from FN to RLD, before going to GCL at different temperatures based upon voltage [32,33]. While the lowest voltage, Fig. 2(a), has a sharp knee, the higher voltages where $\mathrm{FN}$ is an important contributor have more rounded knees, especially Figs. 2(c) and 2(d). This is a desirable quality in a Miram curve, since experimental data exhibit soft knees that have proven difficult to model from first principles [32,33]. Moreover, these Miram curves go to the GCL limit, which is higher than the standard CL limit. Figure 2(c) is at $\bar{V}=\bar{V}_{N}$; we can see the range of influence the nexus has upon the Miram curve by comparing RLD to GTF-not only does the nexus involve the FN baseline contribution, but the knee itself is shifted to a slightly higher temper- ature, not evident at the lower voltage in Fig. 2(b). Moreover, the onset of space charge is more gradual. Figure 2(d) is above the nexus voltage and shows deviates even further from traditional Miram curves. In fact, at sufficiently high voltage, FE would already be at the CL limit and increasing temperature would add enough initial velocity to require a GCL model instead.

Figures 1 and 2 show that nexuses can predict when parameters will have an enormous impact on solutions to emission problems, even multiple orders of magnitude away from the nexus. Knowing the conditions for all nexuses informs researchers when a single simple theory will give accurate results or, if a more complete theory is needed, which physics must be included. To this end, we construct several phase plots over parameters of interest to experiment and device design. Since nexus theory is simple to expand, we incorporate mobility to represent collisions and an external resistance to represent the overall system circuit-solving appropriate combinations of asymptotes from (18) simultaneously gives the permutations for nexuses of second to fifth order.

Figures 3-6 show phase plots for various combinations of axes using the zero temperature CL limit of (14) for clarity. Since five physical parameters-pressure (inverse mobility), temperature, resistance, voltage, and gap distance-describe the complete diode parameter space, two-dimensional (2D) plots require fixing three of the parameters. To construct these plots, we first plotted every second-order nexus line, assigning the regions based on the appropriate limits of large and small values of each axis. For instance, when $\bar{V}$ is an axis, OL 


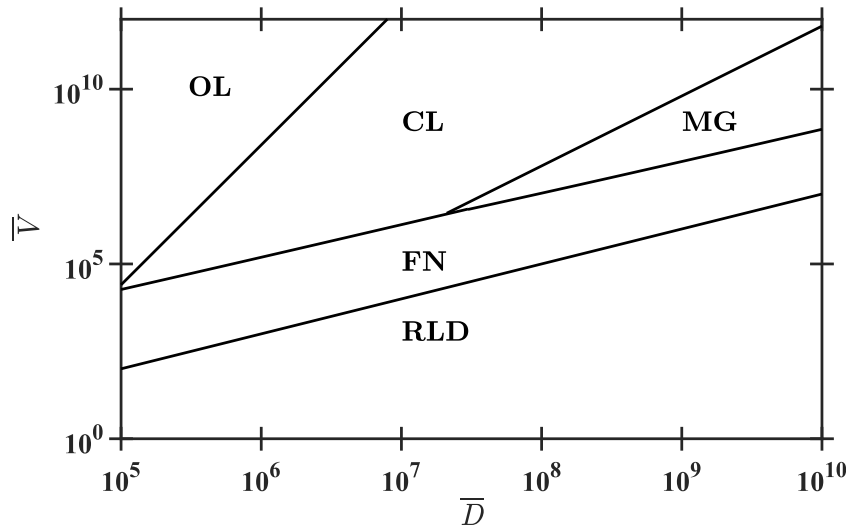

FIG. 3. Phase plot showing the conditions for various emission regimes, Ohm's law (OL), Child-Langmuir (CL), Mott-Gurney (MG), Fowler-Nordheim (FN), and Richardson-Laue-Dushman (RLD) as a function of dimensionless voltage $\bar{V}$ and dimensionless gap distance $\bar{D}$ at fixed dimensionless electron mobility $\bar{\mu}=7 \times$ $10^{3}$, dimensionless external series resistance $\bar{R}=10^{8}$, and dimensionless temperature $\bar{T}=10^{-3}$. Each black line represents a secondorder nexus curve between two regimes; the intersections between the MG-CL and FN-CL lines is a third-order nexus, FN-MG-CL.

occurs at very high $\bar{V}$ [36]; likewise, RLD occupies high $\bar{T}$ and MG low $\bar{\mu}$ (high pressure) [20]. As regions are assigned, spurious sections of the second-order nexus lines need to be trimmed. Since a point where multiple asymptotes intersect is a third- or higher-order nexus point, the lines terminate at that higher-order nexus point. Figure 5 gives an example of the phenomenon; the curve separating RLD from the other four regions is piecewise continuous, consisting of the second-order nexus curves between RLD and FN, MG, CL, and OL as $\bar{V}$ and $\bar{T}$ increase. Additionally, Fig. 5 has the OL

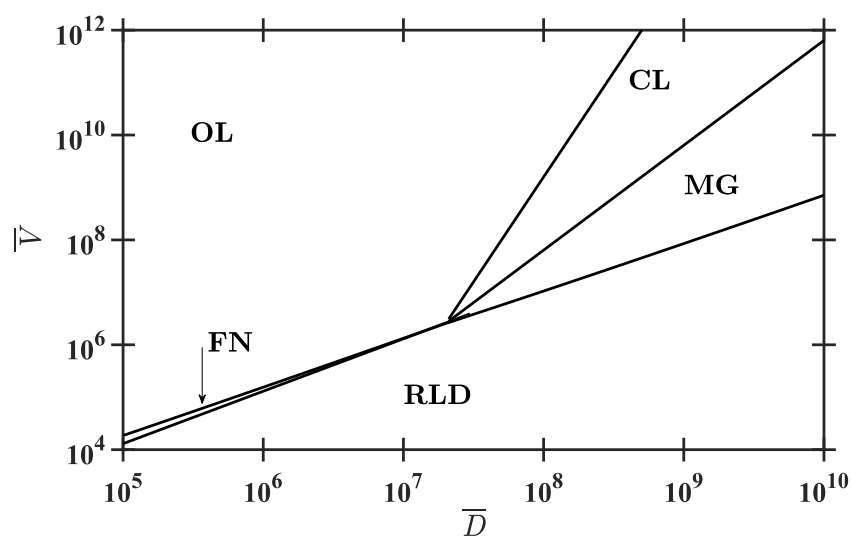

FIG. 4. Phase plot showing the conditions for various emission regimes, Ohm's law (OL), Child-Langmuir (CL), Mott-Gurney (MG), Fowler-Nordheim (FN), and Richardson-Laue-Dushman (RLD) as a function of dimensionless voltage $\bar{V}$ and dimensionless gap distance $\bar{D}$ at fixed dimensionless electron mobility $\bar{\mu}=$ $7 \times 10^{3}$, dimensionless external series resistance $\bar{R}=4 \times 10^{11}$, and dimensionless temperature $\bar{T}=0.12$. Each black line represents a second-order nexus curve between two regimes; the fixed parameters were chosen such that all lines intersect, leading to a fifth-order nexus.

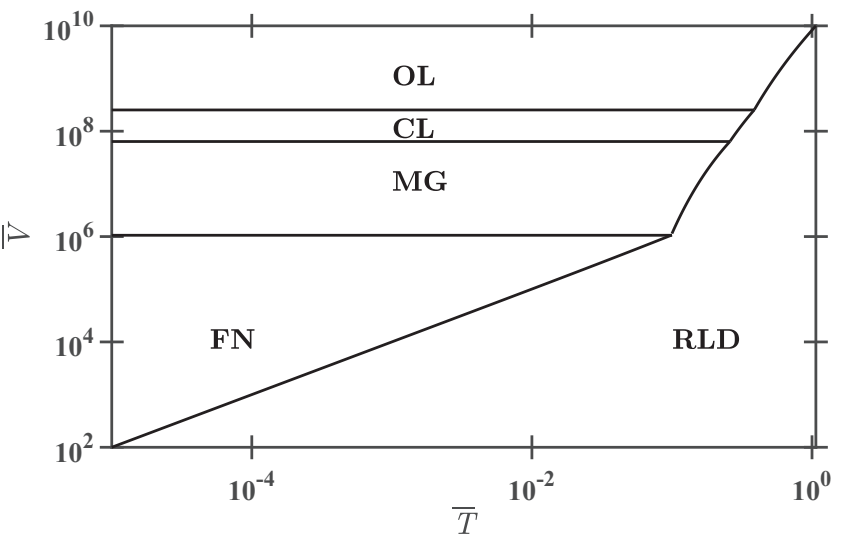

FIG. 5. Phase plot showing the conditions for various emission regimes, Ohm's law (OL), Child-Langmuir (CL), Mott-Gurney (MG), Fowler-Nordheim (FN), and Richardson-Laue-Dushman (RLD) as a function of dimensionless voltage $\bar{V}$ and dimensionless temperature $\bar{T}$ at fixed dimensionless electron mobility $\bar{\mu}=7 \times 10^{2}$, dimensionless external series resistance $\bar{R}=10^{10}$, and dimensionless gap distance $\bar{D}=10^{7}$. Each black line represents a second-order nexus curve between two regimes; several third-order nexuses exist where three asymptotic solutions intersect.

to MG or FN nexus curves absent, because the FN to MG and MG to CL transitions occur at lower voltages than their respective transitions to OL and are superseded.

For these phase plots, the axis limits and fixed parameters were selected to display as many regions and transitions as possible - one may use (5) to translate the dimensionless parameters into physical units for specific devices of interest. Since the complete parameter space is five dimensional, these 2D plots provide a starting point and ideas for possible interpretations of nexus theory. As an additional note, mobility can be difficult to ascertain because although it primarily

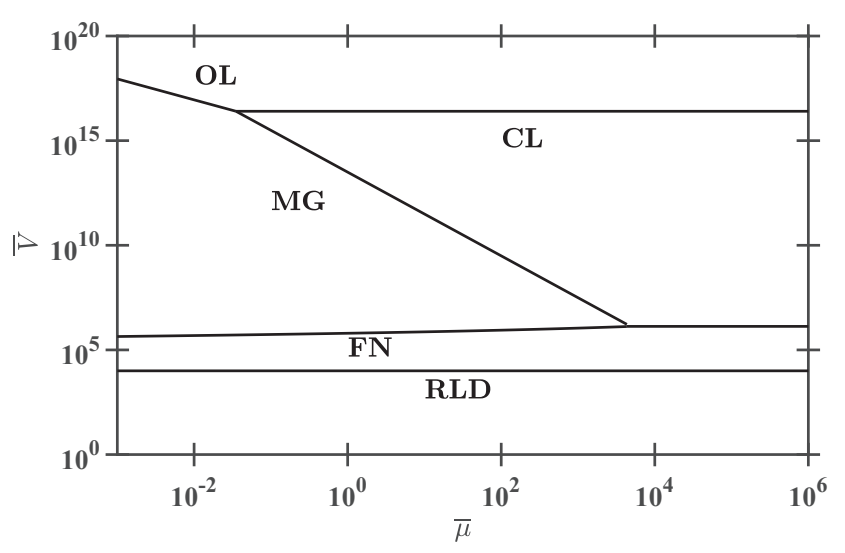

FIG. 6. Phase plot showing the conditions for various emission regimes, Ohm's law (OL), Child-Langmuir (CL), Mott-Gurney (MG), Fowler-Nordheim (FN), and Richardson-Laue-Dushman (RLD) as a function of dimensionless voltage $\bar{V}$ and dimensionless electron mobility $\bar{\mu}$ at fixed dimensionless gap distance $\bar{D}=10^{7}$, dimensionless external series resistance $\bar{R}=10^{6}$, and dimensionless temperature $\bar{T}=10^{-3}$. Each black line represents a second-order nexus curve between two regimes; two third-order nexuses are shown where these curves intersect. 
depends upon pressure, the electric field also contributes-in our previous papers [20,36], we assumed $E=V / D$.

Figure 3 explores the $\bar{V}-\bar{D}$ parameter space with $\bar{\mu}=700$, $\bar{T}=10^{-3}$, and $\bar{R}=10^{8}$; for an emission area of $1 \mu \mathrm{m} \times 1 \mu \mathrm{m}$ and a tungsten diode with surface treatment to lower the work function to $1 \mathrm{eV}$ as in [57], (5) gives the physical values of these parameters as $10^{3} \mathrm{~cm}^{2} /(\mathrm{V} \mathrm{s}), 11.6 \mathrm{~K}$, and $8.1 \mathrm{M} \Omega$. Interpreting mobility is more challenging and different for every gas; for nitrogen, Ref. [58] gives a model $\mu \sqrt{E P}=3.3 \times 10^{6}$ with cgs units for $\mu$ and $E$ and pressure $P$ in Torr. Using $E=10^{4} \mathrm{~V} / \mathrm{cm}$ (reasonable, since this example translates the starting point for the $V$ axis at $5.8 \mathrm{~V}$ and the $D$ axis at $85 \mu \mathrm{m}$ ) yields 1089 Torr. This example demonstrates the process for translating phase plots for physical experiments to understand relevance. Obviously, other experiments may use different materials and focus on a different phase space than presented here. A third-order nexus occurs at the FN-MG-CL intersection. Additional nexuses are present at lower $\bar{D}$ (OL-CL-FN) and at much higher $\bar{D}$ (FN-MG-RLD) than shown; clearly, the limits of parameter space have a huge influence upon which transitions and nexuses manifest. Indeed, as long as each region has a viable parameter space-for instance, drastically increasing $\bar{R}$ would suppress CL and MG-there will exist higher-order nexuses at the intersections of all adjacent regions. We chose the higher range of $\bar{D}$ to display the MG regime.

Constructing an analogous phase plot to design the voltage range for a particular gap distance such as in Fig. 1 would require ignoring the OL and MG regions $(\bar{R} \rightarrow 0$ and $\bar{\mu} \rightarrow$ $\infty$ ) and focusing on $\bar{D}$ from $10^{-1}$ to $10^{5}$. Since each individual physical diode has different scaling constants in (5) and unique fixed parameters, we stress that these phase plots are intended to show the range of possible behaviors. The process above may be followed to create phase plots to suit individual experimental conditions. The conditions for these nexuses and regimes of relevance may be manipulated by changing one of the parameters, such as changing $\bar{R}$ to move the OL-CL boundary.

Figure 4 increases the order of the nexus point in the $\bar{V}-\bar{D}$ space by changing $\bar{R}$ and $\bar{T}$ to $4 \times 10^{11}$ and 0.12 , respectively, to collapse all three third-order nexuses into a single, fifth-order nexus point. For context, with the same emission area and material used for Fig. 3, (5) gives these parameters as $R=32 \mathrm{G} \Omega$ and $T=1400 \mathrm{~K}$. By further customization, parameter space morphologies may be tailored to include or exclude mechanisms from physically occurring, or to leverage transition physics for emission enhancement, such as measuring a field enhanced Miram curve device. Note that the FN region has nearly disappeared in Fig. 4. From Figs. 1 and 2, the parameters must be at least an order of magnitude from a transition for an asymptotic model to be reasonably accurate (cf. Fig. 1). Practically speaking, pure FE will not occur for the conditions in Fig. 4, although enhancement due to FE will still be present, so the physics must still be included in any full solution near to the dwindling FN region. Reducing $\bar{R}$ would revive the FN region, while degrading the fifth-order nexus point to fourth order.

Since this study incorporates temperature into electron emission compared to our previous work $[20,36]$, the $\bar{V}-\bar{T}$ parameter space is particularly relevant. Figure 5 shows that varying $\bar{V}$ and $\bar{T}$ instead of $\bar{D}$ at fixed $\bar{\mu}=700, \bar{D}=10^{7}$, and $\bar{R}=10^{10}$ leads to three third-order nexuses within the chosen $\bar{V}-\bar{T}$ space: FN-MG-RLD, MG-CL-RLD, and CL-OL-RLD. Using the example diode established in Fig. 3, these dimensionless parameters would be translated into the physical values $\mu=10^{3} \mathrm{~cm}^{2} /(\mathrm{V} \mathrm{s})$ (1089 Torr), $D=8.5 \mathrm{~mm}$, and $R=$ $810 \mathrm{M} \Omega$. The fixed parameters were chosen to enlarge each region on the phase plot; however, one could easily eliminate the MG or CL regions by modifying $\bar{\mu}$ and/or $\bar{R}$-each project and device can benefit from a custom nexus phase plot if multiphysics are suspected. Relegating the influence of RLD to higher temperatures than shown in Fig. 5 requires changing $\bar{D}$, the primary limiting parameter, as inferred from (18).

The $\bar{V}-\bar{\mu}$ parameter space is also important: $\bar{\mu}$ varies inversely with pressure. Considering $\bar{V}$ and $\bar{\mu}$ in Fig. 6 at constant $\bar{T}=10^{-3}, \bar{D}=10^{7}$, and $\bar{R}=10^{6}$ shows third-order nexuses for FN-MG-CL and MG-CL-OL. Again, for our example diode, these are $T=11.6 \mathrm{~K}, D=8.5 \mathrm{~mm}$, and $R=$ $81 \mathrm{k} \Omega$. This temperature would be useful for a researcher interested in cryogenics-for instance, cryogenic field-emission scanning electron microscopy (cryo-FESEM), which deals with temperatures as low as $123 \mathrm{~K}$ [59] or $88 \mathrm{~K}$ [60]. With a higher work-function material (say, tungsten at $\Phi=4.5 \mathrm{eV}$ ), $\bar{T}=10^{-3}$ becomes $T=52.2 \mathrm{~K}$, meaning a researcher would only need to slightly adjust other parameters to make Fig. 6 relevant for cryo-FESEM. Interestingly, for the particular $\bar{T}$ considered, RLD only intersects with FN in this parameter space; a higher $\bar{T}$ would easily eliminate $\mathrm{FN}$ and make the RLD-MG and RLD-CL transitions important instead. Most individual experiments consider a much smaller subset of phase space; however, plotting a much larger space demonstrates transitions that may otherwise be neglected. For instance, in Fig. 6 a researcher examining $\bar{V}>10^{7}$ and $\bar{\mu}>$ $10^{4}$ would not encounter the FN regime, but Figs. 1 and 2 demonstrate that the FN to CL nexus may influence the solution even two orders of magnitude away in $\bar{V}$.

It is instructive to consider the critical temperature below which emission transitions solely between FN and CL. In the GTF model, emission begins to transition between thermal and field at $T_{\min } \equiv(\hbar e E) /\left(k_{B} 2 \sqrt{2 m \Phi} t(y)\right)$ and becomes purely thermal at $T_{\max }=(\hbar e E) /\left(k_{B} \pi \sqrt{m \Phi y}\right)$ [35]. For a copper electrode with $\Phi=4.5 \mathrm{eV}, D=100 \mathrm{~nm}$, and $V=$ $1 \mathrm{~V}$, ignoring space charge $E=10^{8} \mathrm{~V} / \mathrm{m}, T_{\min }=53.3 \mathrm{~K}$, and $T_{\max }=165.6 \mathrm{~K}$. Thus, most applications require accounting for thermionic emission to fully capture emission behavior (although the cryogenic cases mentioned above would fall within this range). At room temperature, $T=300 \mathrm{~K}$, the transition from RLD to FN occurs from $2.21 \times 10^{8} \mathrm{~V} / \mathrm{m}<E<$ $5.68 \times 10^{8} \mathrm{~V} / \mathrm{m}$. For $E$ above this range, emission becomes purely $\mathrm{FE}$, although such high $E$ risks damaging the cathode. These examples demonstrate the usefulness of the full theory and asymptotic solutions to an experimentalist over a wide range of $T$ and $E$.

\section{CONCLUSION}

We have derived an emission model including the physics (and respective models) of FE (FN), TE (RLD), and vacuum SCLE with (GCL) and without (CL) initial injection velocity and characterized device conditions requiring the exact so- 
lution and those where appropriate asymptotic solutions are sufficient. Moreover, while certain theories examine the transition between FE and TE [21,26-31], others FE and SCLE $[3,4,21,24,25]$, and others TE and SCLE [32,33], we present here a single theory capable of capturing all these transitions.

The exact solution exhibits several morphologies depending upon system parameters $\bar{D}$ and $\bar{T}$. Transitions could include all the physics (RLD to FN to CL) or neglect certain mechanisms. For low $\bar{T}$, only FN and CL occur; at high $\bar{T}$, GCL transformed directly into CL. Varying $\bar{T}$ gives a modified, field enhanced Miram curve based on this combined physics and shows that $\bar{J}$ from the full solution begins with a baseline FN contribution, transitions to $\bar{J}_{\mathrm{GCL}}$ sooner, and results in a softened knee, a desirable quality in a Miram curve $[32,33]$. The Miram curve typically considers just TE and SCLE; including the GTF emission model incorporates FE into the theory, too. Figure 5 shows that FE can contribute as much as TE at lower $\bar{T}$, quantified in Figs. 1(d)-1(f).

We also demonstrated that the asymptotic nature of nexus theory easily allows the addition (or removal) of mechanisms while retaining predictive power. Once a nexus is of third order (called a triple point) and defined by three physical parameters, such as $\bar{V}, \bar{D}$, and $\bar{T}$, higher-order nexuses may be found by simply equating a new asymptotic theory to the existing nexus, motivating the possible need to incorporate more physics within the exact solution if physical diode parameters are near the nexus. For instance, a triple point was found for $\bar{V}, \bar{D}$, and dimensionless electron mobility $\bar{\mu}$, representing FE and SCLE at both vacuum and pressure [20]. Combining that nexus with (15) by specifying $\bar{T}_{N}=\bar{V}_{N} / \bar{D}_{N}$ gives a fourth-order nexus where FE, TE, and SCLE for vacuum and general pressure agree asymptotically. Introducing resistance to this fourth-order nexus gives a fifth-order nexus that includes Ohm's law, as shown in Fig. 4. Higher-order nexuses could include surface roughness [61], geometric parameters such as curvature [62], AC voltage modulation [63], and magnetic fields [64]. These nexuses will be followed by rigorous derivations of exact solutions, such as those devised for the transitions between microscale and macroscale emission (PL) [9-12]. Future experiments may examine the transitions between these mechanisms under various design conditions and the feasibility of achieving the theoretically predicted field enhanced Miram curve.

\section{ACKNOWLEDGMENTS}

This material is based upon work supported by the Air Force Office of Scientific Research under Award No. FA955018-1-0218. A.M.D. gratefully acknowledges funding from a Purdue doctoral fellowship. C.R.D. gratefully acknowledges funding from a Purdue Summer Undergraduate Research Fellowship (SURF).

\section{APPENDIX}

These supplemental equations for clarification of (4) from the general-thermal-field model use the same definitions found throughout this paper, except where noted [27,30,35]:

$$
\begin{gathered}
y=\frac{\sqrt{4 Q F}}{\Phi}, \\
v(y)=1-\frac{y^{2}}{3}[3-\ln (y)], \quad t(y)=1+\frac{y^{2}}{9}[1-\ln (y)], \\
\phi=(1-y) \Phi \\
T_{\min }=\frac{\hbar F}{2 k_{B} t(y) \sqrt{2 m \Phi}} \\
T_{\max }=\frac{\hbar F}{\pi k_{B} \sqrt{m \Phi y}} .
\end{gathered}
$$

For $T<T_{\min }$,

$$
\beta_{F}=\frac{2 t(y)}{\hbar F} \sqrt{2 m \Phi}, \quad s=\frac{4 v(y) \sqrt{2 m \Phi^{3}}}{3 \hbar F} .
$$

For $T>T_{\max }$,

$$
\beta_{F}=\frac{\pi \sqrt{m \Phi y}}{\hbar F}, \quad s=\frac{\pi \sqrt{m \Phi y}}{\hbar F} \phi .
$$

In the intermediate case, $n=1$, thus $\beta_{F}=\beta_{T}$, and

$$
s=\beta_{T}\left(E_{m}+\frac{\theta\left(E_{m}\right)}{\beta_{F}\left(E_{m}\right)}-\mu^{*}\right),
$$

where $\mu^{*}$ is the Fermi energy and $E_{m}$ is solved from

$$
\beta_{T}=\beta_{F}\left(E_{m}\right),
$$

where

$$
\begin{aligned}
\beta_{F}(E) \approx & \frac{1}{\phi}\left[B_{q} z+C_{\mathrm{FN}}(1-z)\right. \\
& \left.+3\left(2 B_{\mathrm{FN}}-B_{q}-C_{\mathrm{FN}}\right) z(1-z)\right],
\end{aligned}
$$

and

$$
\begin{aligned}
\theta(\mu+z \phi)= & B_{\mathrm{FN}}-C_{\mathrm{FN}} z+z^{2}\left[\left(C_{\mathrm{FN}}-C_{q}\right)(2-z)\right. \\
& \left.-\left(B_{\mathrm{FN}}-B_{q}\right)(3-2 z)\right],
\end{aligned}
$$

with

$$
\begin{aligned}
B_{\mathrm{FN}} & =\frac{4 v(y) \sqrt{2 m \Phi^{3}}}{3 \hbar F}, \quad C_{\mathrm{FN}}=\frac{2 t(y)}{\hbar F} \sqrt{2 m \Phi}, \\
B_{q} & =C_{q}=\frac{\pi \sqrt{2 m}}{\hbar} \phi\left(\frac{Q}{F^{3}}\right)^{1 / 4} .
\end{aligned}
$$

Finally,

$$
N(n, s)=n \int_{-\infty}^{\infty} \frac{\ln \left[1+e^{n(k-s)}\right]}{1+e^{k}} d k .
$$


[1] P. Zhang, A. Valfells, L. K. Ang, J. W. Luginsland, and Y. Y. Lau, 100 years of the physics of diodes, Appl. Phys. Rev. 4, 011304 (2017).

[2] K. L. Jensen, A tutorial on electron sources, IEEE Trans. Plasma Sci. 46, 1881 (2018).

[3] Y. Y. Lau, Y. Liu, and R. K. Parker, Electron emission: from the Fowler-Nordheim relation to the Child-Langmuir law, Phys. Plasmas 1, 2082 (1994).

[4] Y. Feng and J. P. Verboncoeur, Transition from FowlerNordheim field emission to space charge limited current density. Phys. Plasmas 13, 073105 (2006).

[5] W. S. Boyle and P. Kisliuk, Departure from Paschen's law of breakdown in gases, Phys. Rev. 97, 255 (1955).

[6] D. B. Go and A. Venkattraman, Microscale gas breakdown ion-enhanced field emission and the modified Paschen's curve, J. Phys. D 47, 503001 (2014).

[7] R. H. Fowler and L. Nordheim, Electron emission in intense electric fields, Proc. R. Soc. London, Ser. A 119, 173 (1928).

[8] A. Venkattraman and A. A. Alexeenko, Scaling law for direct current field emission-driven microscale gas breakdown, Phys. Plasmas 19, 123515 (2012).

[9] A. M. Loveless and A. L. Garner, Scaling laws for gas breakdown for nanoscale to microscale gaps at atmospheric pressure, Appl. Phys. Lett. 108, 234103 (2016).

[10] A. M. Loveless and A. L. Garner, Generalization of microdischarge scaling laws for all gases at atmospheric pressure, IEEE Trans. Plasma Sci. 45, 574 (2017).

[11] A. M. Loveless and A. L. Garner, A universal theory for gas breakdown from microscale to the classical Paschen law, Phys. Plasmas 24, 113522 (2017).

[12] A. M. Loveless, G. Meng, Q. Ying, F. Wu, K. Wang, Y. Cheng, and A. L. Garner, The transition to Paschen's law for microscale gas breakdown at subatomic pressures, Sci. Rep. 9, 5669 (2019).

[13] F. Paschen, Über die zum Funkenübergang in luft, Wasserstoff und Kohlensäure bei verschiedenen Drucken erforderliche potentialdiffenrenz, Ann. Phys. 273, 69 (1889).

[14] G. Meng, X. Gao, A. M. Loveless, C. Dong, D. Zhang, K. Wang B. Zhu, Y. Chen, and A. L. Garner, Demonstration of field emission driven microscale gas breakdown for pulsed voltages using in-situ optical imaging, Phys. Plasmas 25, 082116 (2018).

[15] G. Meng, Q. Ying, A. M. Loveless, F. Wu, K. Wang, Y. Fu, A. L. Garner, and Y. Cheng, Spatio-temporal dynamics of pulsed gas breakdown in microgaps, Phys. Plasmas 26, 014506 (2019).

[16] J. R. Haase and D. B. Go, Analysis of thermionic and thermofield emission in microscale gas discharges, J. Phys. D: Appl. Phys. 49, 055206 (2016).

[17] A. Venkattraman, Generalized criterion for thermo-field emission driven electrical breakdown of gases, Appl. Phys. Lett. 104, 194101 (2014).

[18] M. U. Lee, J. Lee, J. K. Lee, and G. S. Yun, Extended scaling and Paschen law for micro-sized radiofrequency plasma breakdown, Plasma Sources Sci. Technol. 26, 034003 (2017).

[19] M. U. Lee, J. Lee, G. S. Yun, and J. K.Scalings and universality for high-frequency excited high-pressure argon microplasma, Eur. Phys. J. D 71, 94 (2017).
[20] A. M. Darr, A. M. Loveless, and A. L. Garner, Unification of field emission and space charge limited emission with collisions, Appl. Phys. Lett. 114, 014103 (2019).

[21] P. Y. Chen, T. C. Cheng, J. H. Tsai, and Y. L. Shao, Space charge effects in field emission nanodevices, Nanotechnol. 20, 405202 (2009).

[22] R. Bogue, MEMS sensors: past, present, and future, Sens. Rev. 27, 7 (2007).

[23] H. G. Craighead, Nanoelectromechanical Systems, Science 290, 1532 (2000).

[24] R. G. Forbes, Exact analysis of surface field reduction due to field-emitted vacuum space charge, in parallel-plane geometry, using simple dimensionless equations, J. Appl. Phys. 104, 084303 (2008).

[25] A. Rokhlenko, K. L. Jensen, and J. L. Lebowitz, Space charge effects in field emission: one dimensional theory, J. Appl. Phys. 107, 014904 (2010).

[26] C. R. Crowell, Richardson constant and tunneling effective mass for thermionic and thermionic-field emission in Schottky barrier diodes, Solid-State Electron. 12, 55 (1969).

[27] K. L. Jensen and M. Cahay, General thermal-field emission equation, Appl. Phys. Lett. 88, 154105 (2006).

[28] S. He, J. Scharer, J. Booske, and S. Sengele, One-dimensional combined field and thermionic emission model and comparison with experimental results, J. Vac. Sci. Technol. B 26, 770 (2008).

[29] M. S. Benilov and L. G. Benilova, Field to thermo-field to thermionic electron emission: a practical guide to evaluation and electron emission from arc cathodes, J. Appl. Phys. 114, 063307 (2013)

[30] K. L. Jensen, M. McDonald, O. Chubenko, J. R. Harris, D. A. Shiffler, N. A. Moody, J. J. Petillo, and A. J. Jensen, Thermal-field and photoemission from meso- and micro-scale features: effects of screening and roughness on characterization and simulation, J. Appl. Phys. 125, 234303 (2019).

[31] G. Segev, Y. Rosenwaks, and A. Kribus, Loss mechanisms and back surface field effect in photon enhanced thermionic emission converters, J. Appl. Phys. 114, 044505 (2013).

[32] Y. Wang, J. Wang, W. Liu, L. Li, Y. Wang, and X. Zhang, Correlation between emission behavior and surface features of scandate cathodes, IEEE Trans. Electron Devices 56, 776 (2009).

[33] W. Liu, Y. Wang, J. Wang, Y. Wang, and B. Vancil, Emission characteristics of nanosized Scandia-doped dispenser cathodes in open electron-gun structures, IEEE Trans. Electron Devices 58, 1241 (2011).

[34] K. L. Jensen, P. G. O'Shea, and D. W. Feldman, Generalized electron emission model for field, thermal, and photoemission, Appl. Phys. Lett. 81, 3867 (2002).

[35] K. L. Jensen, General formulation of thermal field and photoinduced electron emission, J. Appl. Phys. 102, 024911 (2007).

[36] S. D. Dynako, A. M. Darr, and A. L. Garner, Incorporating resistance into the transition from field emission to space charge limited emission with collisions, IEEE J. Electron Devices Soc. 7, 650 (2019)

[37] K. Xie, S. A. Hartz, V. M. Ayres, B. W. Jacobs, R. M Ronningen, A. F. Zeller, T. Baumann, and M. A. Tupta, Thermionic field emission in GaN nanoFET Schottky barriers, Mater. Res. Express 2, 015003 (2015) 
[38] K. A. A. Khalid, T. J. Leong, and K. Mohamed, Review on thermionic energy converters, IEEE Trans. Electron Devices 63, 2231 (2016).

[39] A. Varonides, Combined thermionic and field emission reverse current for ideal graphene/n-Si Schottky contacts in a modified Landauer formalism, Phys. Status Solidi C 13, 1040 (2016).

[40] O. W. Richardson and A. F. A. Young, The thermionic workfunctions and photo-electric thresholds of the alkali metals, Proc. R. Soc. London, Ser. A 107, 377 (1925).

[41] D. Perello, S. C. Lim, S. J. Chae, I. Lee, M. J. Kim, Y. H. Lee, and M. Yun, Thermionic field emission transport in carbon nanotubes transistors, ACS Nano 5, 1756 (2011).

[42] M.-C. Kan, J.-L. Huang, J. C. Sun, K.-H. Chen, and B.-S. Yau, Thermionic emission of amorphous diamond and field emission of carbon nanotubes, Carbon 41, 2839 (2003).

[43] F. A. M. Köck, J. M. Garguilo, B. Brown, and R. J. Nemanich, Enhanced low-temperature thermionic field emission from surface-treated N-doped diamond films, Diam. Relat. Mater. 11, 774 (2002).

[44] M. Jang and J. Lee, Analysis of Schottky barrier height in small contacts using a thermionic-field emission model, ETRI J. 24, 455 (2002).

[45] X. He, J. Scharer, J. Booske, and S. Sengele, Modeling of cold emission cathode by inclusion of combined field and thermionic emission processes, J. Appl. Phys. 102, 056107 (2007).

[46] F. A. M. Köck and R. J. Nemanich, Field penetration and its contribution to field enhanced thermionic electron emission from nanocrystalline diamond films, Diam. Relat. Mater. 15, 2006 (2006)

[47] F. Jin, Y. Liu, and C. M. Day, Thermionic emission from carbon nanotubes with a thin layer of low work function barium strontium oxide surface coating, Appl. Phys. Lett. 88, 163116 (2006).

[48] J. M. Shannon, Thermionic-field emission through silicon Schottky barriers at room temperature, Solid-State Electron. 20, 869 (1977).

[49] P. Liu, Q. Sun, F. Zhu, K. Liu, K. Jiang, L. Liu, Q. Li, and S. Fan, Measuring the work function of carbon nanotubes with thermionic method, Nano Lett. 8, 647 (2008).

[50] M. E. Kiziroglou, X. Li, A. A. Zhukov, P. A. J. de Groot, and C. H. de Groot, Thermionic field emission at electrodeposited Ni-Si Schottky barriers, Solid-State Electron. 52, 1032 (2008).

[51] K. L. Jensen, A quantum dipole-modified work function for a simplified electron emission barrier, J. Appl. Phys. 111, 054916 (2012)
[52] E. L. Murphy and R. H. Good, Jr., Thermionic emission, field emission, and the transition region, Phys. Rev. 102, 1464 (1956).

[53] R. G. Forbes, Simple good approximations for the special elliptic functions in standard Fowler-Nordheim tunneling theory for a Shottky-Nordheim barrier, Appl. Phys. Lett. 89, 113122 (2006).

[54] A. M. Darr and A. L. Garner, A coordinate system invariant formulation for space-charge limited current in vacuum, Appl. Phys. Lett. 115, 054101 (2019).

[55] J. W. Luginsland, A. Valfells, and Y. Y. Lau, Effects of a series resistor on electron emission from a field emitter, Appl. Phys. Lett. 69, 2770 (1996).

[56] R. R. Puri, D. Biswas, and R. Kumar, Generalization of ChildLangmuir law for non-zero injection velocities in a planar diode, Phys. Plasmas 11, 1178 (2004).

[57] S. H. Chou, J. Voss, A. Vojvodic, R. T. Howe, and F. AbildPedersen, DFT study of atomically-modified alkali-earth metal oxide films on tungsten, J. Phys. Chem. C 118, 11303 (2014).

[58] N. M. Zubarev and S. N. Ivanov, Mechanism of runaway electron generation at gas pressures from a few atmospheres to several tons of atmospheres, Plasma Phys. Rep. 44, 445 (2018).

[59] P. Echlin, Low temperature scanning electron microscopy: a review, J. Microsc. 112, 47 (1978).

[60] Y. Chen, V. E. Centonze, A. Verkhovsky, and G. G. Borisy, Imaging of cytoskeletal elements by low-temperature high resolution scanning electron microscopy, J. Microsc. 179, 67 (1995).

[61] R. S. Brayfield, II, A. J. Fairbanks, A. M. Loveless, S. Gao, A. Dhanabal, W. Li, C. Darr, W. Wu, and A. L. Garner, The impact of cathode surface roughness and multiple breakdown events on microscale gas breakdown at atmospheric pressure, J. Appl. Phys. 125, 203302 (2019).

[62] Z. B. Zhu and L. K. Ang, Space charge limited current emission for a sharp tip, Phys. Plasmas 22, 052106 (2015).

[63] G. Herink, L. Wimmer, and C. Ropers, Field emission at terahertz frequencies: AC-tunneling and ultrafast carrier dynamics, New J. Phys. 16, 123005 (2014).

[64] A. Rokhlenko and J. L. Lebowitz, Space-Charge-Limited 2D Electron Flow Between Two Flat Electrodes in a Strong Magnetic Field, Phys. Rev. Lett. 91, 085002 (2003).

Correction: The previously published Figures 5 and 6 contained incorrect labels on the abscissa and have been replaced. 